\title{
EPIDEMIOLOGIA DAS PARASITOSES INTESTINAIS EM CRIANÇAS DE CRECHES DE RIO VERDE-GO
}

\author{
INTESTINAL PARASITOSIS EPIDEMIOLOGY IN CHILDREN FROM \\ A CHILD DAY CARE CENTERS IN RIO VERDE-GO, BRAZIL
}

Marilúcia F. Zaiden; Branca M. de O. Santos²; Maria Aparecida T. Cano²; lucif A. Nascif Júnior ${ }^{3}$

\begin{abstract}
${ }^{1}$ Bióloga; Mestre em Promoção de Saúde pela Universidade de Franca/SP (UNIFRAN- SP); Docente da FESURV - Universidade de Rio Verde - GO. ${ }^{2}$ Docentes do Curso de Mestrado em Promoção de Saúde (UNIFRAN- SP); Enfermeiras, Docentes Aposentadas da Escola de Enfermagem de Ribeirão Preto-USP. ${ }^{3}$ Médico Veterinário, Docente do Curso de Mestrado em Promoção de Saúde (UNIFRAN- SP) CorRespondÊnCIA: Branca M. de O. Santos. Rua Imbuia, 25. Jardim Recreio. CEP 14040-200. Ribeirão Preto-SP. Tel: $3630-7877$. brancamosantos@yahoo.com.br
\end{abstract}

Zaiden MF; Santos BMO; Cano MAT; Nascif Júnior IA. Epidemiologia das parasitoses intestinais em crianças de creches de Rio Verde - GO. Medicina (Ribeirão Preto) 2008; 41 (2): 182-7.

RESUMO: O estudo teve como objetivos avaliar os fatores de risco e a prevalência das parasitoses intestinais em 276 crianças de 0 a 6 anos de creches municipais de Rio Verde-GO. As amostras foram obtidas no primeiro trimestre de 2005 e submetidas aos métodos diagnósticos de Hoffman, Pons e Janer e de Faust e as informações acerca dos fatores de risco foram obtidas junto às mães e/ou responsáveis através de um questionário. A maior prevalência foi em crianças na faixa etária de quatro anos, do sexo feminino, procedentes do próprio bairro onde a creche estava localizada, cujos responsáveis possuíam escolaridade fundamental incompleta. O protozoário Giardia lamblia foi o mais prevalente, seguido por Entamoeba coli e associação entre ambos, com diferença estatística significante entre as creches. O contato com solo foi outro fator de risco significativo para prevalência de parasitoses intestinais. Os resultados apontam para a importância da melhoria das condições socioeconômicas e sanitárias e do saneamento básico por meio de ações a serem implementadas em programas de promoção de saúde.

Descritores: Parasitos. Criança. Creches. Epidemiologia. Prevalência.

\section{1- INTRODUÇÃO}

As parasitoses intestinais provocadas por protozoários e helmintos são infestações que podem desencadear alterações no estado físico, psicossomático e social, interferindo diretamente na qualidade de vida de seus portadores, principalmente em crianças de classes sociais mais baixas, com precárias condições sanitárias, maus hábitos de higiene, em situação de desnutrição e em locais de aglomerações tais como creches, escolas, asilos e orfanatos, pela facilidade de contaminação e disseminação. A presença desses agentes etiológicos pode aumentar sobremaneira, tanto em quantidade quanto em qualidade, conforme as alterações ambientais sejam favoráveis à presença e manutenção concomitante de diversos tipos de parasitas, resultando no poliparasitismo, situação debilitante para o indivíduo ${ }^{1-6}$.

O equacionamento deste problema de saúde pública esbarra na necessidade de conhecimento da realidade e dos fatores de risco que favorecem o surgimento, a manutenção e a propagação desses agentes, dentre os quais se destacam as condições de moradia e saneamento básico da população exposta, os hábitos alimentares, de higiene pessoal, de contato com o solo e a presença de reservatórios no local. 
Diante dessas considerações é que se realizou este estudo com o objetivo de avaliar os fatores de risco e a prevalência das parasitoses intestinais em crianças de zero a seis anos de creches municipais de Rio Verde-GO.

\section{2- MATERIAL E MÉTODOS}

Este estudo de caráter descritivo-analítico foi realizado em quatro creches municipais de Rio Verde-GO, com perfis socioeconômicos diferenciados, sendo uma localizada na região central da cidade $(\mathrm{C} 1)$ e três em áreas periféricas carentes $(\mathrm{C} 2, \mathrm{C} 3$ e $\mathrm{C} 4)$, selecionadas em função do número de crianças atendidas, na faixa etária de zero a seis anos, com permanência integral (das $7 \mathrm{~h}$ às $17 \mathrm{~h}$ ) no local, e de prérequisitos como a autorização de acesso às creches $\mathrm{e}$ às crianças e/ou responsáveis. Foram mantidos contatos prévios com a Secretaria Municipal de Saúde para a obtenção de autorização dos procedimentos clínicos e laboratoriais das amostras de fezes, com vistas à identificação dos parasitos e, subseqüente, tratamento. Na Secretaria Municipal de Educação obteve-se autorização para acesso às creches. O estudo foi também aprovado pelo Comitê de Ética em Pesquisa da Universidade de Franca, sob o $\mathrm{n}^{\circ}$ 128118/ 2005.

Para subsidiar a avaliação dos resultados obtidos das amostras fecais utilizou-se um questionário para respostas das mães e/ou responsáveis, contendo dados de identificação da criança e questões sobre alguns fatores de risco para ocorrência de parasitoses, como condições de moradia e saneamento básico, hábitos higiênicos da criança, casos diagnosticados anteriormente na criança e/ou familiares e alguma informação verbalizada pelas mães e/ou responsáveis pela criança.

Os critérios de inclusão para que a criança participasse do estudo foram: não estar utilizando nenhum antiparasitário durante o período da coleta; concordância da mãe e/ou responsável através da assinatura do Termo de Consentimento Livre e Esclarecido e preenchimento do questionário de coleta das informações epidemiológicas pelo responsável pela criança.

Foram realizadas reuniões com as mães e/ou responsáveis, diretoras e monitoras das creches com a finalidade de prestar informações sobre as parasitoses intestinais e suas conseqüências à saúde e oferecer orientações sobre a coleta e o recolhimento das amostras de fezes.
As amostras foram coletadas durante o primeiro trimestre de 2005, sendo uma de cada criança. Procedia-se a coleta da porção central das fezes, em frascos coletores esterilizados, com o auxílio de uma espátula de madeira e luvas de procedimento descartáveis. Para as crianças que usavam fralda, as fezes foram coletadas da própria fralda. As amostras obtidas foram submetidas à análise, no máximo, oito horas após a coleta, sendo mantidas em geladeira durante esse tempo. A análise das mesmas foi realizada por dois métodos laboratoriais de diagnóstico: Método de Faust (centrífugo-flutuação em solução de sulfato de zinco) e o de Sedimentação Espontânea conhecido como Método de Lutz ou de Hoffman, Pons e Janer ${ }^{7}$. O segundo método foi utilizado apenas nas amostras negativas ao primeiro, pelo fato de o mesmo ser mais econômico e rápido. Os resultados foram confrontados com os dos fatores de risco. Foram calculadas a média e o desvio padrão e aplicados o teste do quiquadrado e ANOVA, sendo considerados significativos valores de $p<0,05$.

\section{3- RESULTADOS}

Das 276 crianças que participaram do estudo, $49(17,8 \%)$ eram da $\mathrm{C} 1,36(13,0 \%)$ da $\mathrm{C} 2,66(23,9 \%)$ da C3 e $125(45,3 \%)$ da C4. A C1, por ter localização central, atendia em sua maioria $(71,4 \%)$ crianças de outros bairros. Nas demais, a grande maioria das crianças provinha do mesmo bairro da creche.

A média de idade das crianças foi de três anos, das quais $149(54,0 \%)$ eram do sexo feminino, sendo que $63(22,8 \%)$ apresentaram resultados positivos de infecção por protozoários e/ou helmintos. Das 127 $(46,0 \%)$ crianças do sexo masculino, $47(17,0 \%)$ estavam infectadas. A prevalência total foi de $39,9 \%$ (110 crianças). Não foram encontradas diferenças significativas para as variáveis idade e sexo.

Os resultados da Tabela I demonstram uma maior prevalência de protozooses em relação às helmintoses. As amostras positivas para Giardia lamblia e Entamoeba coli foram 21,4\% e 12,0\%, respectivamente. O poliparasitismo ocorreu em $12(4,4 \%)$ e o monoparasitismo em 98 (35,5\%) das crianças.

Na distribuição por creche observa-se a predominância de G. lamblia nas crianças de todas as creches $(\mathrm{C} 1,36,7 \%$; $\mathrm{C} 2,27,8 \%$; $\mathrm{C} 3,21,2 \%$ e C4, 13,6\%).

A E. coli apresentou maior prevalência na C4 (16,0\%). Os resultados da associação de $G$. lamblia/E. coli foram mais prevalentes na $\mathrm{C} 2(5,5 \%)$ 
e C3 (4,6\%) . A prevalência de Ascaris lumbricoides foi maior na $\mathrm{C} 3(1,5 \%)$, não ocorrendo casos nas $\mathrm{C} 1$ e C2. A associação E. coli/Endolimax nana, foi identificada apenas na C3 (3,0\%). A variação entre as creches não foi estatisticamente significativa, porém, quando se analisou os tipos de parasitas encontrados em relação à cada creche observou-se diferenças significativa $\left(X^{2}(21)=33,779 ; p=0,038\right)$.

Observou-se que em $80,5 \%$ dos casos, a mãe era a responsável pela criança. Quanto à escolaridade $71,0 \%$ tinham ensino fundamental incompleto e $7,6 \%$ eram analfabetos.

As crianças moravam em casas de alvenaria em $70,7 \%$ dos casos e $24,6 \%$ em construções alternativas (taipa não revestida, taipa revestida ou de madeira). O número de cômodos das casas variou de um a seis. Não foram encontradas diferenças estatísticas entre os casos de parasitoses e o tipo de residência e nem em relação ao número de cômodos.

Em $76,4 \%$ das casas o fornecimento de água era realizado por rede pública e $22,8 \%$ por cisternas. Em 64,9\% dos casos o destino dos dejetos humanos era realizado em esgotamento sanitário, 33,3\% em fossas e $0,7 \%$ em esgoto a céu aberto. A coleta do lixo pelo sistema público de limpeza ocorreu em $98,2 \%$ dos casos. Em 0,4\% o lixo era enterrado, em 0,7\% era queimado e em $0,7 \%$ disposto a céu aberto. Essas variáveis não demonstraram significância estatística.

Quanto aos hábitos higiênicos das crianças, verificou-se que $92,4 \%$ faziam uso de chuveiro e $4 \%$ de banhos de bacia. As mãos eram lavadas antes das refeições por $93,1 \%$ das crianças e após o uso do sanitário por $85,5 \%$ delas.
Em relação aos hábitos alimentares, 43,8\% dos responsáveis referiram que a criança tinha o hábito de ingerir verduras cruas ou cozidas ou lavadas, $10,1 \%$ cruas ou cozidas e $0,7 \%$ apenas verduras cruas. Não foram encontradas significâncias estatísticas em relação às parasitoses.

O hábito de andar descalço foi encontrado em $72,8 \%$ das crianças, porém não houve diferença estatística significativa. No entanto, $89,5 \%$ das crianças tinham contato com o solo (terra) sob diversas formas e houve diferença significativa $\left(X^{2}(7)=19,819\right.$, $\mathrm{p}=0,006)$.

A presença de reservatórios animais na residência foi variável: $25,7 \%$ referiram presença de cão, $2,5 \%$ de gato, $2,5 \%$ de cão e gato, $0,4 \%$ de cão, gato e outros tipos de animais convivendo diretamente no mesmo ambiente, sem diferença significativa com relação ao número de casos positivos para os parasitos intestinais.

\section{4- DISCUSSÃO}

Os dados relativos à idade das crianças reforçam o fato de encontrarem-se num período da vida cuja incidência de parasitoses é maior, tendo em vista as mudanças em relação à resposta imune aos parasitos e hábitos pessoais, sociais e alimentares, tais como introdução de alimentos crus na dieta, diminuição dos cuidados diretos, maior contato com o solo, com outras crianças e animais domésticos, sendo importante a implementação de medidas preventivas primárias e secundárias. A resposta imuno/dependente aumenta com a idade e exposição ao parasito ${ }^{8,9,10}$.

Tabela I: Distribuição dos protozoários e helmintos entéricos por creche analisada.

\begin{tabular}{|c|c|c|c|c|c|c|c|c|c|c|}
\hline \multirow{2}{*}{ Parasitos } & \multicolumn{2}{|c|}{ Creche 1} & \multicolumn{2}{|c|}{ Creche 2} & \multicolumn{2}{|c|}{ Creche 3} & \multicolumn{2}{|c|}{ Creche 4} & \multirow[b]{2}{*}{$\mathrm{N}^{\mathrm{o}}$} & \multirow{2}{*}{$\begin{array}{c}\text { Total } \\
(\%)\end{array}$} \\
\hline & $\mathrm{N}^{\circ}$ & $(\%)$ & $\mathrm{N}^{\mathrm{o}}$ & $(\%)$ & $\mathrm{N}^{\mathrm{o}}$ & $(\%)$ & $\mathrm{N}^{\mathrm{o}}$ & $(\%)$ & & \\
\hline Giardia lamblia & 18 & $(36,7)$ & 10 & $(27,8)$ & 14 & $(21,2)$ & 17 & $(13,6)$ & 59 & $(21,4)$ \\
\hline Entamoeba coli & 01 & $(2,0)$ & 04 & $(11,1)$ & 08 & $(12,1)$ & 20 & $(16,0)$ & 33 & $(12,0)$ \\
\hline Ascaris lumbricoides & 0 & - & 0 & - & 01 & $(1,5)$ & 01 & $(0,8)$ & 02 & $(0,7)$ \\
\hline Hymenolepis nana & 0 & - & 0 & - & 03 & $(4,6)$ & 01 & $(0,8)$ & 04 & $(1,4)$ \\
\hline G. lamblia/ Endolimax nana & 0 & - & 0 & - & 0 & - & 01 & $(0,8)$ & 01 & $(0,4)$ \\
\hline G. lamblia/E. coli & 0 & - & 02 & $(5,5)$ & 03 & $(4,6)$ & 04 & $(3,2)$ & 09 & $(3,3)$ \\
\hline E. coli/E. nana & 0 & - & 0 & - & 02 & $(3,0)$ & 0 & - & 02 & $(0,7)$ \\
\hline Negativo & 30 & $(61,2)$ & 20 & $(55,6)$ & 35 & $(53,0)$ & 81 & $(64,8)$ & 166 & $(60,1)$ \\
\hline Total & 49 & $(17,8)$ & 36 & $(13,0)$ & 66 & $(23,9)$ & 125 & $(45,3)$ & 276 & $(100,0)$ \\
\hline
\end{tabular}


Apesar de alguns estudos mostrarem uma maior prevalência em crianças do sexo masculino ${ }^{1,4,5,11}$, os resultados desta pesquisa apontaram que a infecção nas crianças do sexo feminino foi mais prevalente, ainda que existam trabalhos que não fazem referência entre as possíveis diferenças de prevalência de parasitoses intestinais em relação ao sexo das crianças $^{12,13,14}$.

O elevado índice de resultados negativos $(60,1 \%)$ foi satisfatório, porém remete à possível influência do ciclo reprodutivo dos parasitos nos resultados, uma vez que foi realizada apenas uma coleta de amostra. A possibilidade de encontrar parasitos nas fezes aumenta pelo exame de amostras múltiplas, em razão da intermitência da passagem de certos parasitos no hospedeiro, da eliminação não uniforme dos ovos de helmintos, dos diferentes estágios dos protozoários e das limitações dos métodos de diagnóstico ${ }^{7}$.

É unânime que os dois protozoários mais prevalentes no estudo ( $G$. lamblia e E. coli), bem como Hymenolepis nana e A. lumbricoides estão relacionados às condições ambientais, falta de hábitos de higiene e de saneamento básico, confinamento em creches e asilos, contaminação do depósito sub-ungueal, ingestão de água e alimentos contaminados, contato com o solo e à precariedade da educação sanitária $^{1,5,8,13,15}$.

Os poliparasitismos $(4,4 \%)$ verificados podem ser justificados pelo fato de as creches receberem crianças de áreas geográficas diferentes, com suas particularidades ambientais de saneamento básico e socioeconômico-culturais que contribuem para maior ou menor prevalência de tipos diferenciados de parasitoses e pelo fato de os parasitos envolvidos apresentarem o mesmo mecanismo de transmissão, atuando como bons indicadores das condições sócio-sanitárias e fornecendo melhor entendimento da epidemiologia das parasitoses ${ }^{12,13,16}$.

A maioria $(80,5 \%)$ das crianças ficava sob a responsabilidade da mãe, o que demonstra um comportamento típico da sociedade brasileira, em que a mesma, na maioria dos casos, se responsabiliza pelos cuidados dos filhos. A despeito da escolaridade dos responsáveis não ter se mostrado significativa na ocorrência de parasitoses intestinais, outros autores apontam esse fator como indicador de maior suscetibilidade às parasitoses ${ }^{4,17,18,19}$. Ele também retrata aspectos da condição socioeconômica da população carente de informações, dentre elas as formas de prevenção das doenças parasitárias que interferem no de- senvolvimento físico, mental e emocional das crianças, alertando para a necessidade de maior investimento no ensino ofertado à população ${ }^{17,18,20}$.

Os resultados de vários estudos apontam para correlação entre as condições de saneamento básico e parasitoses intestinais ${ }^{1,21,22,23}$. No entanto, os resultados encontrados de alguns fatores de risco epidemiológicos, como a qualidade da água, destino dos dejetos humanos e destino do lixo doméstico, não demonstraram significância estatística quando confrontados com a presença de positividade aos parasitas.

A higiene pessoal, como tomar banho todos os dias, lavar as mãos antes das refeições e após a defecação, cortar as unhas, andar calçado, dentre outras medidas básicas, são necessárias para uma boa saúde. Esses fatores são importantes na redução dos riscos de infestação por parasitos entre as crianças ${ }^{2,3,8}$. Porém, a análise dos dados não apresentou diferença significativa com os resultados de parasitoses intestinais encontrados.

Os cuidados com a preparação e a forma de consumo de alimentos também são fatores que podem proteger ou propiciar a ocorrência das parasitoses intestinais, uma vez que a manipulação incorreta dos alimentos pode estar diretamente relacionada à contaminação, sendo que as hortaliças e a carne têm sido consideradas um importante meio de transmissão, principalmente se consumidas in natura ${ }^{10,24,25}$.

Apesar do hábito de andar descalça não ter mostrado diferença estatística significativa, o contato das crianças com o solo representou um fator de risco. Ainda que os parasitos encontrados não tenham transmissão percutânea, a faixa etária das crianças nos remete a ocorrência da infecção através do contato da mão contaminada com terra na boca ${ }^{26}$.

A maior parte dos parasitas encontrados não tem relação com a presença de reservatórios animais convivendo no mesmo ambiente. Porém, não se descarta a importância do convívio de animais constituir um fator de risco para a contaminação por parasitas. A maioria das casas não reserva um local para a evacuação desses animais, além do fato de poderem circular livremente pelas ruas adjacentes às suas casas ficando vulneráveis à contaminação por parasitos através da ingestão de água e alimentos fora do domicílio, podendo também carrear cistos de parasitos em seus pêlos, tornando-se fontes de infecção para seus donos, principalmente as crianças ${ }^{1}$.

Essas considerações demandam a necessidade de cuidados, incluindo desverminação, principalmente 
em cães e gatos filhotes e jovens, preferidos pelas crianças, uma vez que esses podem estar infectados e serem fontes de infecção $0^{3,11,27,28}$.

A disseminação das parasitoses também pode ocorrer através do contato interpessoal com pessoas infectadas que habitam a mesma residência, principalmente em moradias menores que favorecem o confinamento, reforçando a importância da investigação parasitária na população materno-infanti1 ${ }^{17,20}$.

\section{5- CONCLUSÕES}

Os fatores de risco a que as crianças estão expostas podem agravar a situação de parasitoses intestinais. Verificou-se que o tipo de parasitas encontrados pode variar entre crianças de creches localizas em áreas geográficas distintas. Além disso, o contato dessas crianças com o solo contaminado pro- piciou uma maior prevalência de infecções intestinais por parasitos.

Os resultados obtidos neste estudo mostram que ainda há uma prevalência importante de parasitoses intestinais em crianças na faixa etária de zero a seis anos. Isso pode representar um problema de saúde pública, fortalecendo a convicção acerca da importância da prevenção através da melhoria das condições socioeconômicas, de saneamento básico e da educação em saúde.

Conclui-se que as condições higiênico-sanitárias da moradia, das pessoas e do ambiente aos quais as crianças estão submetidas podem contribuir de forma significativa para uma maior prevalência de parasitoses intestinais na faixa etária considerada. Desta forma, a melhoria das condições socioeconômicas e sanitárias e do saneamento básico deveriam ser ações contempladas em programas de promoção de saúde.

Zaiden MF; Santos BMO; Cano MAT; Nascif Júnior IA. Intestinal parasitosis epidemiology in children from a child day care centers in Rio Verde-GO, Brazil. Medicina (Ribeirão Preto) 2008; 41 (2): 182-7.

ABSTRACT: The objectives of study was to evaluate risk factors and prevalence of intestinal parasitosis in 276 children from 0 to 6 years old in four municipal child day care centers from Rio Verde, State of Goiás, Brazil. Samples had been collected in the first trimester of 2005 and submitted to Hoffman, Pons and Janer and Faust diagnostic methods and information concerning risk factors had been gotten, through a form, from mothers and/or the children responsible. The highest prevalence was in female four years children, from the day care centers neighborhood, whose responsible had incomplete basic education. Giardia lamblia was the most prevalent protozoan, followed by Entamoeba coli and association between both, with significant statistics difference between the day care centers. Soil contact was another significant risk factor in he prevalence of intestinal parasitosis. Results indicates that the importance social-economic and sanitary conditions and basic sanitation improvement through the implementation of health promotion actions.

Keywords: Parasites. Child. Day Care Centers. Epidemiology. Prevalence.

\section{REFERÊNCIAS}

1 - Faleiros JMM et al. Ocorrência de enteroparasitoses em alunos da escola pública de ensino fundamental do município de Catanduva (São Paulo, Brasil). Rev Inst Adolfo Lutz 2004; 63 (2): 243-7.

2 - Giraldi N, Vidotto O, Navarro IT, Garcia JL. Enteroparasites prevalence among daycare and elementary school children of municipal schools, Rolandia, PR, Brazil. Rev soc bras. med trop 2001; 34 (4): 385-7.

3 - Gurgel RQ, Cardoso GS, Silva AM, Santos LN, Oliveira RCV. Creche: ambiente expositor ou protetor nas infestações parasitárias intestinais em Aracajú, SE. Rev soc bras. med trop 2005; 38 (3): 267-9.
4 - Machado RC, Marcari EL, Cristante SFV, Carareto CMA. Giardíase e helmintíase em crianças de creches e escolas de $1^{\circ}$ e $2^{\circ}$ graus (públicas e privadas) da cidade de Mirassol (SP, Brasil). Rev soc bras. med trop 1999; 32 (6): 697-704.

5 - Mendoza D et al. Parasitosis intestinales en 4 circulos infantiles de San Miguel del Padrón, Ciudade de La Habana. Rev Cubana,1998. Med trop 2001; 53 (3): 189-93.

6 - Quadros RM, Marques S, Arruda AAR, Delfes PSWR, Medeiros IAA. Parasitas intestinais em centros de educação infantil municipal de Lages, SC (Brasil). Rev soc bras. med trop 2004; 37 (5): 422-3.

7 - De Carli GA. Parasitologia Clínica: seleção de métodos e técnicas de laboratório para o diagnóstico das parasitoses humanas. São Paulo: Atheneu; 2001. 
8 - Bezerra FSM, Oliveira MF, Miranda ALL, Pinheiro MCC, Teles RMA. Incidência de parasitos intestinais em material subungueal e fecal em crianças da Creche Aprisco -Fortaleza, CE. Rev bras anal clin 2003; 35 (1): 39-40.

9 - Bórquez C, Lobato I, Montalvo MT, Marchant P, Martínez, P. Enteroparasitosis en niños escolares del valle de Lluta. Arica - Chile. Parasitol latinoam. 2004; 59 (3-4): 175-8.

10 - Nolla AC, Cantos GA. Prevalência de enteroparasitoses em manipuladores de alimentos, Florianópolis, SC. Rev soc bras med trop 2005; 38 (6): 524-5.

11 - Beck C, Araújo FAP, Olicheski, AT, Breyer AS. Freqüência da infecção por Giardia lamblia (Kunstler, 1882) em cães (Canis familiaris) avaliada pelo método de Faust e cols. (1939) e pela Coloração da Auramina, no município de Canoas, RS (Brasil). Ciênc rural 2005; 35 (1): 126-30.

12 - Menezes AL, Lima VMP, Freitas MTS, Rocha MO, Silva EF, Dolabella SS. Prevalence of intestinal parasites in children from public daycare centers in the city of Belo Horizonte, Minas Gerais, Brazil. Rev inst med trop S. Paulo 2008,50(1):57-9.

13 - Buschini MLT, Pittner E, Czervinski T, Moraes IF, Moreira MM, Sanches HF, Monteiro MC. Spatial distribution of enteroparasites among school children from Guarapuava, State of Paraná, Brazil. Rev bras epidemiol 2007; 10(4): 568-78.

14 - Santos MES, Ogando T, Fonseca BPV, Júnior, CEG, Barçante, JMP. Ocorrência de enteroparasitos em crianças atendidas no Programa de Saúde da Família de uma área de abrangência do município de Vespasiano, Minas Gerais. Rev elet enf 2006, 8(1): 25-9.

15 - Tashima NT, Simões MJS. Enteroparasitic occurrence in fecal samples analyzed at the University of Western São PauloUNIOESTE clinical laboratory, Presidente Prudente, São Paulo State, Brazil. Rev inst med trop São Paulo. 2004; 46 (5): 243-8.

16 - Rocha RS et al. Avaliação da esquistossomose e de outras parasitoses intestinais em crianças do município de Bambuí, Minas Gerais (Brasil). Rev soc bras med trop 2000; 3 (5): 431-6.

17 - Ferreira MU, Ferreira CS, Monteiro CA. Tendência secular das parasitoses intestinais na infância na cidade de São Paulo (1984-1996). Rev saúde pública 2000; 34 (6 Supl): 73-82.
18 - Fortes BPMD, Ortiz Valencia LI, Ribeiro SV, Medronho RA. Modelagem geoestatística da infecção por Ascaris lumbricoides. Cad saúde pública 2004; 20 (3): 727-34.

19 - Núñez FA, Lopez JL, La Cruz AM, Finlay CM. Fatores de riesgo de la infecccion por Giardia lamblia em niños de guardeíras infantiles de ciudad de la Habana, Cuba. Cad saúde pública 2003; 19 (2): 677-82.

20 - Costa-Macedo LM, Rey L. Aleitamento e parasitismo intestinal materno-infantil. Rev soc bras med trop 2000; 33 (4): 371-5.

21 - Fontbonne A, Freese-de-Carvalho E, Acioli MD, Sá GA Cesse EAP. Fatores de risco para poliparasitismo intestinal em uma comunidade indígena de Pernambuco (Brasil). Cad saúde pública 2001; 17 (2): 367-73.

22 - Ferreira GR, Andrade CFS. Alguns aspectos socioeconômicos relacionados a parasitoses intestinais e avaliação de uma intervenção educativa em escolares de Estiva Gerbi, SP. Rev soc bras med trop 2005; 38(5): 402-5.

23 - Rego RCF, Barreto ML, Killinger CL. O que é lixo afinal? Como pensam mulheres residentes na periferia de um grande centro urbano. Cad saúde pública 2002; 18 (6): 1583-92.

24 - Silva JO, Capuano DM, Takayanagui OM, Giacometti Júnior E. Enteroparasitose e onicomicoses em manipuladores de alimentos do município de Ribeirão Preto, SP (Brasil). Rev bras epidemiol 2005; 8 (4): 385-92.

25 - Soares B, Cantos GA. Qualidade parasitológica e condições higiênico-sanitárias de hortaliças comercializadas na cidade de Florianópolis, Santa Catarina (Brasil). Rev bras epidemiol 2005; 8 (4): 377-84.

26 - Neves DP. Relação parasito-hospedeiro. In: Parasitologia Humana. 10.ed. São Paulo: Athenu, 2000. cap 2, p. 4-9.

27 - Bartmann A, Araújo FAP. Freqüência de Giardia lamblia em cães atendidos em clínicas veterinárias de Porto Alegre, RS (Brasil). Ciênc rural 2004; 34 (4): 1093-6.

28 - Blazius RD, Emerick S, Prophiro, JS, Romão PRT, Silva OS. Ocorrência de protozoários e helmintos em amostras de fezes de cães errantes da Cidade de Itapema, Santa Catarina. Rev soc bras med trop 2005; 38 (1): 73-4.

Recebido para publicação em 14/11/2007.

Aprovado para publicação em 11/06/2008. 